\title{
Switching between anti-tumour necrosis factors: trying to get a handle on a complex issue
}

\section{Ronald $\mathrm{F}$ van Vollenhoven}

\section{The question of switching is not only a question of clinical practice, but has an important scientific dimension as well \\ See linked article, p 893}

\author{
Make sense who may. I switch off. \\ Samuel Beckett (1984)
}

The registration, almost simultaneously, of etanercept and infliximab, followed some years later by that of adalimumab, presented rheumatologists with outstanding therapeutic options to the benefit of many patients. Nevertheless, it also became clear that none of these drugs are effective in all cases, and the question about switching from one anti-tumour necrosis factor (TNF) to another became an important clinical issue. As we wrote 4 years ago in the Annals of the Rheumatic Diseases: "in these situations, [is] there a rationale for prescribing the other TNF $\alpha$ blocker, or [is this] simply a waste of time and money?". ${ }^{1}$ Since that publication, a sizeable number of research articles have investigated this question, and the answers have generally been in the affirmative, implying that, yes, a switch of this type can benefit some patients. ${ }^{2-16}$ However, some reports reached less favourable conclusions, ${ }^{17}{ }^{18}$ and it can rightfully be asserted that none of those published studies, nor any of the many abstracts presented at international meetings on the topic, were controlled, prospective, randomised or blinded.

Perhaps the importance and depth of the issue of switching between anti-TNFs has not been sufficiently appreciated. For one thing, the question of switching is not only a question of clinical practice, but has an important scientific dimension as well: a true demonstration that one anti-TNF agent has efficacy in the same patient who failed to respond to another suggests something potentially important about the pathophysiological process in that individual, leading to interesting possibilities for "bedside-to-bench" research. A good example of this principle was the case report by Buch et al ${ }^{19}$ of a patient with no discernible response to infliximab but an excellent response to etanercept. The authors thought that this observation was consistent with the hypothesis that in this particular patient lymphotoxin played a major role, a hypothesis that was bolstered by the demonstration of lymphotoxin in the patient's synovial tissue.

Switching between anti-TNF agents is also increasingly an issue of regulatory importance, because healthcare administrators in various countries are currently assessing to what extent a second, or even a third, anti-TNF should be reimbursed and under what circumstances. This issue has become particularly relevant now that both rituximab and abatacept (in the US) have received regulatory approval for the specific claim of efficacy in patients who have failed anti-TNFs. None of the three anti-TNFs has a similar claim, and therefore, going strictly by the book, the nonanti-TNF agents would be more appropriate choices for those patients than switching between anti-TNFs. Medicine is, however, a bit more complicated than that, and therapeutic choices are made, for better or for worse, by considering many aspects other than product labels, including prior experiences, perceptions of risk, practical considerations, "gut feeling" and personal preferences. Clearly, more data on switching between anti-TNFs would be important for regulators and scientists, as well as for clinicians.

In this issue of the Annals of the Rheumatic Diseases, Furst et al ${ }^{20}$ present what could be considered a little milestone on this topic: the OPPOSITE Trial (open-label, pilot protocol of patients with rheumatoid arthritis who switch to infliximab after an incomplete response to etanercept), the first randomised controlled trial of switching between two different anti-TNFs. In this study, 28 patients who had an inadequate response to etanercept (as defined by at least 6 swollen and 9 tender joints despite treatment) were randomised either to continue the original treatment or to switch to infliximab (at the usual recommended dosage and frequency.) After 16 weeks, the patients in the "switch" group had better Disease Activity Score using 28 joint counts (DAS28) scores (mean (SD) 4.0 (1.5) vs $5.2(1.6)$ ), and a higher rate of American College of Rheumatology (ACR) 20 (62\% vs 29\%) and of ACR50 $(31 \%$ vs $14 \%)$ responses, and so on, differences that did not, however, achieve statistical significance in most instances (in fact, statistical significance and $p$ values are not even reported in the paper). Additional analyses showing radiological and MRI changes were not very clear. Nonetheless, the aggregate of the results suggests that switching in this group of patients was slightly more effective than continuing the first agent.

These results probably do not come as a surprise to most of us. Having seen the many observational studies attesting to the benefit of switching, and probably having some personal experiences in individual patients, most rheumatologists are rather convinced that switching can be effective. Nonetheless, not all observations, whether at the personal level or from case series, can be taken at face value, and a peculiarity of these types of observations is that they rarely avoid the bias of "regression to the mean". Briefly, this term refers to the following: disease activity tends to fluctuate over time (around the mean), and patients tend to request, and doctors tend to initiate, therapeutic changes at times of higherthan-average disease activity. Because of this, there is a greater than 50-50 likelihood that the patients will start improving after the intervention just by chance, and the bias is that the improvement will be credited to the intervention. We previously pointed out the importance of this effect in assessing the benefits of dose adjustments with biologicals, ${ }^{21}$ and clearly this and similar considerations make it imperative that the question of switching be addressed using a variety of scientific approaches, including the randomised controlled trial.

Furst et $a l^{20}$ are remarkably restrained in their interpretation of the results. They repeatedly emphasise how this was a small trial, they do not test for statistical significance (of the clinical results) and they cautiously avoid drawing any firm conclusions. They end the paper with what seems to be the promise of a larger trial investigating this issue. Nonetheless, inasmuch as this controlled trial is the only one we have at this moment, it seems that the conclusions are rather clear and straightforward. In this group of patients who had disease activity despite etanercept, switching to infliximab seemed to be a better therapeutic option 
than not changing anything at all. Clinicians will probably be interested in knowing how this particular switch would compare with other reasonable options, such as the addition of low-dose oral glucocorticoids, changes in concomitant disease-modifying antirheumatic drug therapy, the use of intra-articular glucocorticoids for treatment of the most inflamed joints and so on. It is unlikely that randomised trials will address those questions. Meanwhile, those interested in the pathophysiology of rheumatoid arthritis and/or the pharmacodynamics of antiTNFs will perhaps once again be intrigued by the observation that in some patients infliximab does work better than etanercept (which is not to say that it could not be the other way around for other patients), suggesting differences in the exact roles of various cytokines and cellular actors in the pathophysiological processes in those patients. However, the fact remains that the most obvious difference between etanercept and infliximab is intravenous versus subcutaneous dosing, and so it is also conceivable that simple pharmacokinetics are sufficient to explain the results of this study, for instance, by achieving more consistent serum levels.

One of the major weaknesses of virtually all published studies on switching between anti-TNFs, including some from our own unit, is that the failure on the first agent is poorly defined. Failure can, in fact, be one of four distinct possible scenarios (fig 1). The first is that a patient does not respond at all to treatment with the first anti-TNF (primary lack of efficacy). For operational purposes, one might have a definition of this lack of response that would allow for some numerical improvement as long as it is not believed to be clinically relevant. The DAS-based European League Against Rheumatism (EULAR) response criteria (good/moderate/no) clearly do allow for some response without considering it relevant (ie, improvement of $<0.6$, or even $0.6-1.2$ if disease activity remains high). Likewise, not even achieving an ACR20 would, in most cases, be considered a rather poor showing for an antiTNF. The second possibility is a secondary loss of efficacy (also referred to as acquired drug resistance ${ }^{22}$ ). This type of failure refers to the situation when a patient initially responds well to treatment with an anti-TNF, but later loses this response. This event may be linked to the development of antibodies against the biological, as has been demonstrated in various studies. ${ }^{23-25}$ Although secondary loss of efficacy is often discussed, a review of our data from the Stockholm TNF $\alpha$ follow-up registry suggested that it remains a rather uncommon event, affecting around $4 \%$ of patients per year. The third possibility is, of course, that a patient has a good response to the first anti-TNF but develops an intolerance to the drug. This could take the form of an infusion reaction to infliximab or injection site reactions to the subcutaneous agents, or various other side effects that may or may not be different for each of the three drugs. In most registries, such events have not been common. The fourth kind of failure is perhaps the most important one to consider in this context: failure by partial efficacy. This may require some explanation. As has been well established, the true responses to anti-TNF agents are not dichotomous but are distributed as a normal curve. $^{26}$ Therefore, one would expect the greatest number of patients to have neither an outstanding response nor a lack of response, but end up somewhere in the middle. Registry data have repeatedly borne this out: in our own registry, approximately $40 \%$ of patients achieve low disease activity or remission 6 months after taking any of the anti-TNFs, and $60 \%$ do not. Of these $60 \%$, about one-third are EULAR non-responders, and the remaining
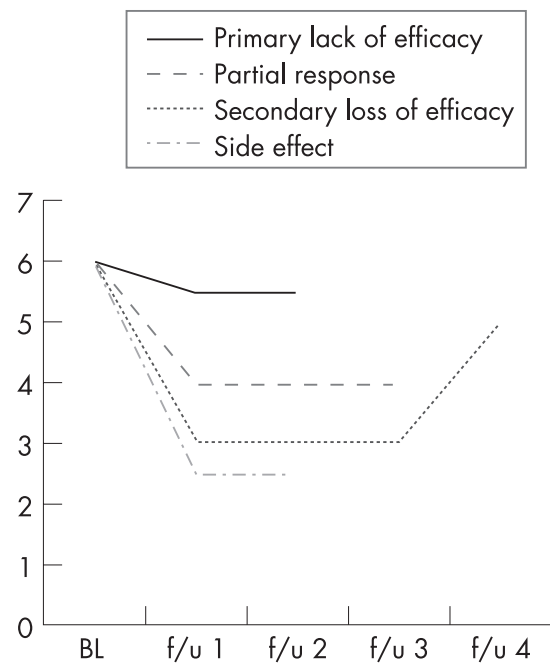

Figure 1 Four different types of failure with antitumour necrosis factors (TNFs). Disease activity is shown at baseline (BL) and four follow-up timepoints (f/u 1-4). Disease activity could, of course, be any of a number of measures, but the values used here would be consistent with it being the Disease Activity Score using joint counts 28. Four hypothetical types of failure are shown: (1) a patient who has no meaningful clinical benefit at all (primary lack of efficacy); (2) a patient who has a measurable and meaningful response, but for whom we could certainly aspire to do better still (partial response); (3) a patient who, after an initial good response, gets worse again (secondary loss of efficacy); and (4) a patient who has a good response but has to discontinue because of a side effect. Each type of anti-TNF failure engenders a different set of questions with respect to switching. two-third are moderate, that is, partial, responders.

Clearly, each failure scenario engenders a different set of questions with respect to switching. If the patient had a primary lack of efficacy, then any meaningful response to the second anti-TNF is a gain. If the patient lost efficacy after initially responding to the first agent, then observing the same efficacy with a second antiTNF is certainly interesting and worthy, even as it raises the question of whether the efficacy of the second drug will also diminish with time. If the patient discontinued the first anti-TNF because of a side effect then any efficacy with another agent is, of course, very welcome. The fourth type of failure is the most tricky. What if the patient responded to the first anti-TNF with a decrease in DAS28 from, say, 6.4 to 4 .l. That would certainly meet the criteria for moderate EULAR response, it would probably also be an ACR50 or so, and it would be much better than nothing at all. On the other hand, such a patient might well be interested in trying something else, to see if one could get better still.

Unfortunately, most studies on observational switching have not defined the type of failure accurately. In their controlled study, Furst et $a l^{20}$ indicate that the patients must have had a response to etanercept (although the magnitude of the response is not prespecified) and a certain minimum level of disease activity must be present for the patient to be enrolled. These requirements could be fulfilled by patients with a secondary loss of efficacy as well as by those with a partial response. That is useful, because in observational studies it is particularly tricky to assess switches in these two situations, because of day-to-day variations in rheumatoid arthritis activity, inaccuracy of the measurements, the impact of comorbidities and non-medical factors, and changes in concomitant medications. Possible approaches are to compare the average disease activity achieved with each anti-TNF (or area under the curve), or to compare the maximally achieved treatment effect with each agent. ${ }^{1}$ Interestingly, the large randomised, controlled trials done with rituximab, abatacept and, recently, tocilizumab, defining the patient population as anti-TNF failures, also did not clearly define the type of failure that each patient had experienced (other than the distinction between failure due to side effects vs other possibilities). For clinicians considering the treatment options for a patient who has failed one anti-TNF, the guidance provided by these trials is therefore also limited in this respect, and it remains a challenge to determine the optimal next step in this situation. 
So where do we stand today on switching between anti-TNFs? Surprisingly, and perhaps discouragingly, we still lack precise answers to some of the most relevant questions that can be asked on this issue. Although scientific and clinical importance have been recognised for some time, they have not led to sufficiently sophisticated studies to settle the issue. Increasingly, there is going to be a regulatory dimension to the matter, which may come to have a major impact on rheumatological practice. We need more and better data. For all these reasons, the contribution by Furst et al ${ }^{20}$ is more than welcome, and the promise of additional controlled trials addressing this issue much appreciated.

Ann Rheum Dis 2007;66:849-851.

doi: 10.1136/ard.2007.069872

Correspondence to: R F van Vollenhoven, Karolinska University Hospital, Stockholm 17176, Sweden; ronald.van.vollenhoven@ki.se

Accepted 27 March 2007

Competing interests: None declared.

\section{REFERENCES}

1 Van Vollenhoven RF, Harju A, Brannemark S, Klareskog L. Treatment with infliximab (Remicade) in patients who failed etanercept (Enbrel) or vice versa: data from the STURE registry showing that switching TNF $\alpha$ blockers can make sense. Ann Rheum Dis 2003;62:1195-8.

2 Brocq O, Plubel Y, Breuil V, Grisot C, Flory P, Mousnier A, et al. Switch étanercept-infliximab dans la polyarthrite rhumatoïde- 14 patients sur 131 traités par anti-TNF $\alpha$. Presse Med 2002;31:1836-9

3 Ang HT, Helfgott S. Do the clinical responses and complications following etanercept or infliximab therapy predict similar outcomes with the other tumor necrosis factor-alpha antagonists in patients with rheumatoid arthritis? J Rheumato/ 2003:30:2315-18.

4 Favalli EG, Arreghini M, Arnoldi C, Panni B, Marchesoni A, Tosi S, et al. Anti-tumor necrosis factor alpha switching in rheumatoid arthritis and juvenile chronic arthritis. Arthritis Rheum 2004;51:301-2.
5 Hansen KE, Hildebrand JP, Genovese MC, Cush JJ Patel S, Cooley DA, et al. The efficacy of switching from etanercept to infliximab in patients with rheumatoid arthritis. J Rheumatol 2004;31:1098-102.

6 Gomez-Puerta JA, Sanmarti R, Rodriguez-Cros JR Canete JD. Etanercept is effective in patients with rheumatoid arthritis with no response to infliximab therapy. Ann Rheum Dis 2004;63:896

7 Haraoui B, Keystone EC, Thorne JC, Pope JE Chen I, Asare CG, et al. Clinical outcomes of patients with rheumatoid arthritis after switching from infliximab to etanercept. J Rheumatol 2004;31:2356-9.

8 Wick MC, Ernestam S, Lindblad S, Bratt J, Klareskog L, van Vollenhoven RF. Adalimumab (Humira) restores clinical response in patients with secondary loss of efficacy from infliximab (Remicade) or etanercept (Enbrel): results from the STURE registry at Karolinska University Hospital. Scand J Rheumatol 2005:34:353-8.

9 Bennett AN, Peterson P, Zain A, Grumley J, Panayi G, Kirkham B. Adalimumab in clinical practice. Outcome in 70 rheumatoid arthritis patients, including comparison of patients with and without previous anti-TNF exposure. Rheumatology (Oxford) 2005;44:1026-31

10 Delaunay C, Farrenq V, Marini-Portugal A Cohen JD, Chevalier X, Claudepierre P. Infliximab to etanercept switch in patients with spondyloarthropathies and psoriatic arthritis: preliminary data. J Rheumatol 2005;32:2183-5.

11 Cohen G, Courvoisier N, Cohen JD, Zaltni S, Sany J, Combe $B$. The efficiency of switching from infliximab to etanercept and vice-versa in patients with rheumatoid arthritis. Clin Exp Rheumato 2005;23:795-800.

12 Gomez-Reino JJ, Carmona L, BIOBADASER Group. Switching TNF antagonists in patients with chronic arthritis: an observational study of 488 patients over a four-year period. Arthritis Res Ther 2006;8:R29

13 Nikas SN, Voulgari PV, Alamanos Y, Papadopoulos CG, Venetsanopoulou Al Georgiadis AN, et al. Efficacy and safety of switching from infliximab to adalimumab: a comparative controlled study. Ann Rheum Dis 2006;65:257-60.

14 Hyrich KL, Lunt M, Watson KD, Symmons DP Silman AJ. British Society for Rheumatology Biologics Register. Outcomes after switching from one anti-tumor necrosis factor alpha agent to a second anti-tumor necrosis factor alpha agent in patients with rheumatoid arthritis: results from a large UK national cohort study, Arthritis Rheum 2007; 56:13-20

15 lannone F, Trotta F, Monteccuco C, Giacomelli R, Galeazzi M, Matucci-Cerinic M, et al. Etanercept maintains the clinical benefit achieved by infliximab in patients with rheumatoid arthritis who discontinued infliximab because of side effects. Ann Rheum Dis 2007;66:249-52

16 Hiardem E, Ostergaard M, Pødenphant J, Tarp U Andersen LS, Bing J, et al. Do rheumatoid arthritis patients in clinical practice benefit from switching from infliximab to a second tumor necrosis factor alpha inhibitor? Ann Rheum Dis. Published Online First 27 March 2007. doi: ard.2006.054742v1.

17 Yazici $Y$, Erkan D. Do etanercept-naive patients with rheumatoid arthritis respond better to infliximab than patients for whom etanercept has failed? Ann Rheum Dis 2004;63:607-8.

18 Solau-Gervais E, Laxenaire N, Cortet B, Dubucquoi S, Duquesnoy B, Flipo RM. Lack of efficacy of a third tumour necrosis factor alpha antagonist after failure of a soluble receptor and a monoclonal antibody. Rheumatology (Oxford) 2006;45:1121-4.

19 Buch MH, Conaghan PG, Quinn MA, Bingham SJ, Veale D, Emery $P$. True infliximab resistance in rheumatoid arthritis: a role for lymphotoxin alpha? Ann Rheum Dis 2004:63:1344-6.

20 Furst DE, Gaylis N, Bray V, Olech E, Yocum D, Ritter J, et al. Open-label, pilot protocol of patients with rheumatoid arthritis who switch to infliximab after an incomplete response to etanercept: the OPPOSITE study. Ann Rheum Dis 2007:66:893-9.

21 van Vollenhoven RF, Brannemark S, Klareskog L. Dosage escalation of infliximab in clinical practice: a registry-based study suggesting that improvements seen after infliximab dosage escalation are largely explained by a regressionlike effect. Ann Rheum Dis 2004;63:426-30

22 Finckh A, Simard JF, Gabay C, Guerne PA. SCQM physicians. Evidence for differential acquired drug resistance to anti-tumour necrosis factor agents in rheumatoid arthritis. Ann Rheum Dis 2006;65:746-52

23 Wolbink GJ, Vis $M$, Lems W, Voskuyl AE, de Groot E, Nurmohamed MT, et al. Development of antiinfliximab antibodies and relationship to clinica response in patients with rheumatoid arthritis. Arthritis Rheum 2006;54:711-15.

24 Bendizen K, Geborek P, Svenson M, Larsson L, Kapetanovic MC, Saxne T. Individualized monitoring of drug bioavailability and immunogenicity in rheumatoid arthritis patients treated with the tumor necrosis factor alpha inhibitor infliximab. Arthritis Rheum 2006;54:3782-9.

25 Bartelds GM, Wiibrandts CA, Nurmohamed MT, Stapel S, Lems WF, Aarden L, et al. Clinical response to adalimumab: the relationship with antiadalimumab antibodies and serum adalimumab concentrations in rheumatoid arthritis. Ann Rheum Dis Published Online First 9 March 2007. doi, ard 2006.065615v2.

26 van Vollenhoven RF, Klareskog L. Clinical responses to tumor necrosis factor $\alpha$ antagonists do not show a bimodal distribution. Data from the Stockholm tumor necrosis factor $\alpha$ registry. Arthritis Rheum 2003:48:1500-3. 